\title{
Analytical Performance of Closed Loop Power Control Quantized under Fast Fading for CDMA Techniques
}

\author{
S.Nourizadeh, T.Jeans and R.Tafazolli \\ CCSR University of Surrey \\ Guildford GU2 7XH, UK \\ Tel: $(+44) .1483 .686 .001$ \\ Fax: (+44).1483.686.011 \\ Email:S.Nourizadeh@surrey.ac.uk
}

\begin{abstract}
The performance of SIR-based Closed Loop power control (CLPC) is analytically analysed. The evaluation work has been carried out using the standard deviation of the power control error (PCE) as the performance metric. A non-linear control theory method is applied to the feedback system under fast fading. An analytical expression of the CLPC under fast fading is also produced. Finally a quantized-step size power control algorithm, replacing the hard limiter is considered. The proposed method is found to work considerably better for high speed MS as well as being a powerful tool to optimise the loop performance.
\end{abstract}

\section{Introduction}

There has been considerable interest lately in using CodeDivision Multiple Access (CDMA) technology to improve the capacity of cellular telephone systems. The capacity of the system in such a multiple access scheme depends heavily on the Multiple Access Interference inherent in a CDMA based cellular structure. Open and Closed Loop Power Control schemes are therefore used to enhance the capacity. Since the fast fading is mitigated via the closed-loop scheme, it is of primary interest to be able to evaluate the performance of the latter, depending on parameters such as the mobile speed and the channel variations. Traditionally, the performance of the closed loop is obtained by simulation, a very time consuming procedure [1]. The aim of this research is to analytically evaluate the performance of the loop under fast fading conditions, so that results can be obtained by a closed formula. Since it is found from both simulation and experimental results that the Power Control Error (PCE) follows a normal distribution if expressed in $\mathrm{dBs}$ [2], the standard deviation (std) of the PCE is adopted as the criterion for the closed loop performance. The first section of this paper expresses the analytical expression of two-feedback methods system under fading, proposed by [3]. The second section applies and proposes the analytical expression of the PCE under fast fading (Rayleigh channel). The results (expressed as the PCE std) obtained from the analytical methods are compared to those obtained from the simulation [4] in the third section. Finally a novel technique is introduced which consists of replacing the hard limiter by a quantizer. Interesting results about the performance of the algorithm with respect to the input range of the quantizer; number of bits used for quantization and the loop delay are obtained.

\section{Mathematical analysis of Closed Loop}

In CDMA-based cellular systems such as UMTS/IMT2000, the CLPC performance varies with different vehicular speeds, propagation channels and delays. In reverse link CLPC (Figure 1), the BS measures the received mean SIR (Signal to Interference Ratio) over the period $T_{P}$, and compares it with the target SIR. It then transmits a power control command (in Figure 1 noted +/1) to the mobile station for a power increase or decrease.

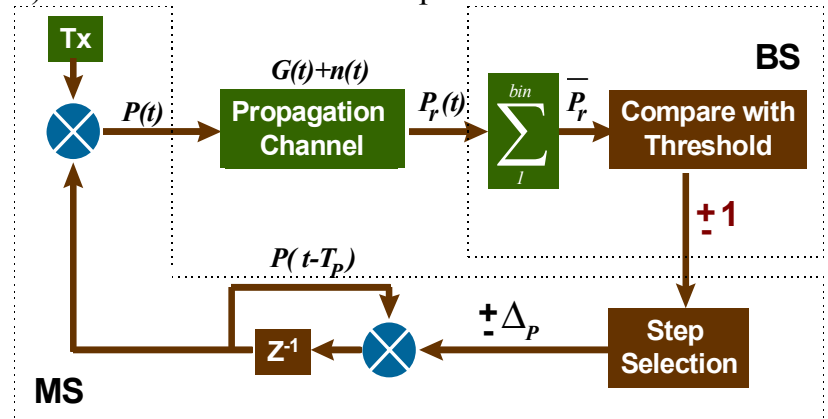

Figure 1: Conventional Closed Loop Power Control Model

[3] studied this problem under slow fading such as shadowing and deduced a modified figure (Figure 2) so as to apply non-linear control theory to the CLPC.

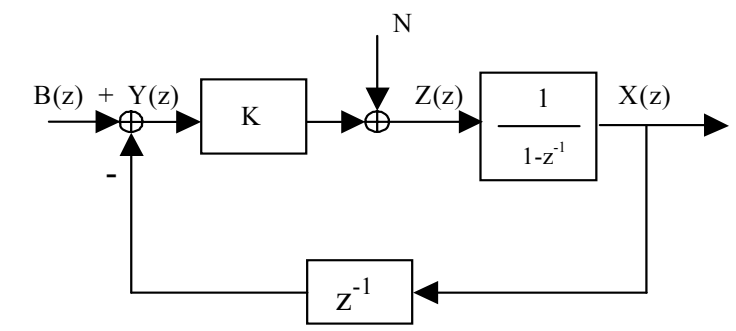

Figure 2: Modified CLPC representation

The performance of the system is analyzed through the Power Control Error (PCE) and expressed in $\mathrm{dB}$ as:

$$
\begin{array}{ll}
Y(n)=\Gamma^{\text {tar }}-A(n)-X(n)+I & \text { Eq } 1 \\
X(n+1)=X(n)+\Delta * \operatorname{sign}[Y(n)] & \text { Eq } 2
\end{array}
$$

where $I, A, X, \Delta$ and $I^{\text {tar }}$ represent respectively, the interference (assumed constant), the fading, the transmit power, the step size and the SIR target. To facilitate the analysis this sequence is defined:

$$
B(n)=-A(n-1)+I+\Gamma^{\text {tar }}
$$


[3] applied the statistical linearisation method to the Eq.2, replacing the non-linear element with the equivalent gain $K$, to the output of which is added an uncorrelated signal (represented by $\mathrm{N}$ in Figure 2). The relation between the $\mathrm{PCE}$ and the gain $K$ is given in Eq.4:

$\sigma_{Y}=\frac{\Delta}{K} \sqrt{\frac{2}{\pi}}$

From the Figure 2, [3] proposed two methods to calculate the PCE, the Spectrum Integration Method (SIM) and the Lyapunov Equation Method (LEM), which consist of solving simultaneously Eq.4 and the equation calculated from each method. SIM assumes that the spectrum of the channel $\mathrm{P}_{\mathrm{B}}$ is known and deduces the transfer functions $H_{Y B}$ and $H_{Y N}$ (Figure 2)

$\sigma_{Y}^{2}=\frac{1}{2 \pi} \int_{-\infty}^{\infty}\left|H_{Y B}\left(e^{j \omega}\right)\right|^{2} P_{B}(\omega) d \omega+\left.\frac{1}{2 \pi} \int_{-\infty}^{\infty} H_{Y N}\left(e^{j \omega}\right)\right|^{2} P_{N}(\omega) d \omega$

Eq 5

$\mathrm{P}_{\mathrm{N}}$ represents the spectrum of the noise $N$. The LEM, on the contrary, operates in time domain and therefore the autocorrelation function of the channel $B$ is required. The standard deviation of the PCE $\sigma_{y}$ is then calculated via Eq.6

$\sigma_{y}^{2}=\frac{2(1-a-b)}{K^{2}(1-a)} \sigma_{x}^{2}+\sigma_{N}^{2}$

Eq 6

The parameters $a$ and $b$ are used only for simplification, $\alpha$ is a parameter that controls the spatial decorrelation of the fading and $N$ is a zero mean Gaussian random process.

$a=-\alpha(1-K)$

$b=1-K+\alpha$

The reader is referred to [3] on obtaining Eq.6. For both methods, the solution $\sigma_{y}$ will be the intersection between the two curves defined by the Eq. $4 \&$ Eq.5 (or Eq.6), as $K$ takes values from zero to two. [3] solves this problem for slow fading such as Shadowing by assuming the variable $A$, therefore $B$ (Eq.3), to have a lognormal distribution as expressed in [5].

\section{CLPC under Rayleigh fading}

In [3], the case where the channel follows a Rayleigh distribution was done semi-analytically. To express a full analytical expression of the CLPC under a fast fading channel has been proven to be not so simple [1]. One reason is that in the proposed model it is very hard to include the autocorrelation of the Rayleigh channel known as the Jakes model, expressed in Eq.7.

$R_{\mu}(t)=J_{o}\left(2 \pi * f_{m} t\right)$

Eq 7

Instead we have concentrated our efforts in the Gaussian power spectral density given by [6]

$S_{\mu}(f)=\frac{\sigma_{\mu}{ }^{2}}{\sqrt{2 \pi} \sigma_{c}} e^{-\frac{f^{2}}{2 \sigma_{c}{ }^{2}}}$

where $\sigma_{\mu}^{2}$ is the variance of the Rayleigh variable, $f$ is the maximum doppler frequency and $\sigma_{c}$ is a parameter that is related to the 3-dB cut-off frequency $f_{c}$ of $S_{\mu}(f)$ according to

$f_{c}=\sigma_{c} \sqrt{2 \ln 2}$

Eq 9
The inverse Fourier transform of Eq.8 gives the corresponding autocorrelation function

$R_{\mu}(t)=\sigma_{\mu}{ }^{2} e^{-\left(\pi \sigma_{c} t\right)^{2}}$

Eq 10

The latter model corresponds to a wave incidence scenario where the signal energy is concentrated in two distinct angular regions, where the angle spread corresponds to the variance of the gaussian shape and the mean angle corresponds to the offset of the Gaussian shape [7].

Lyapunov Method: As it was mentioned before, the Lyapunov method is a time domain process and for that reason the time autocorrelation function of the Rayleigh channel is required. By applying Eq.10 to our closed loop model, where in the Eq. $1 A$ now represents the fast fading and, its autocorrelation is given by Eq.11.

$R(n)=\sigma_{A}^{2} e^{-\left(\pi \sigma_{c} n T\right)^{2}}$

Eq 11

$\sigma_{A}$ is the standard deviation of the Rayleigh signal. $T$ is the sampling period, which is the length of a PCG (Power Control Group, [3]) for the system and $\sigma_{c}$ is a parameter expressed as:

$\sigma_{c}=\frac{f}{\sqrt{2 \ln 2}}=\frac{f_{c} \times v}{c \times \sqrt{2 \ln 2}}$

Eq 12

From Eq.11, we can propose Eq.13, where the parameter $\alpha$ can be identified easily before being inserted to the Eq. 6 via the parameters $a$ and $b$.

$R(n)=\sigma_{A}^{2} \alpha^{|n|}$

Eq 13

Spectrum Integration Method: From the z-transform of the autocovariance of $R(n)$ (Eq.13), an equivalent expression for the Rayleigh power spectrum is obtained:

$P_{B}(\omega)=\sigma_{A}^{2} \frac{\left(1-\alpha^{2}\right)}{1+\alpha^{2}-2 \alpha \cos \omega}$

Eq 14

By including the power spectrum $\mathrm{P}_{\mathrm{B}}$ (Eq.14) into Eq.5, the Spectrum Integration Method will also propose a solution for fast fading. As in the Shadowing case [3], the systems defined by (Eq.4 \& Eq.5 or Eq.6), can now be solved numerically for the Rayleigh channel. For a particular power control step size $\Delta$ and MS speed, the solution to each system will be given by the intersection of the two curves corresponding to a particular value of $K$.

\section{Analytical Performance of CLPC}

In order to verify the results from both techniques under fast fading, we have also simulated the conventional CLPC (shown in the Figure 1) based on the UMTS standard, where the power control period (or PCG) chosen is $0.667 \mathrm{~ms}$ and the step size is fixed to $1 \mathrm{~dB}$ [4]. By setting the latter parameters, according to the UMTS standard, the Lyapunov and the Spectrum Integration Method results are also produced. As mentioned before, the performance of the system is analyzed through the standard deviation of the PCE $\sigma_{y}$. The simulation assumptions and results are identical to [4].

The results of all three methods are represented in the Figure 3. Results from the two analytical methods 
(Lyapunov \& Spectrum Integration) follow those obtained by simulations based on the UMTS standards values.

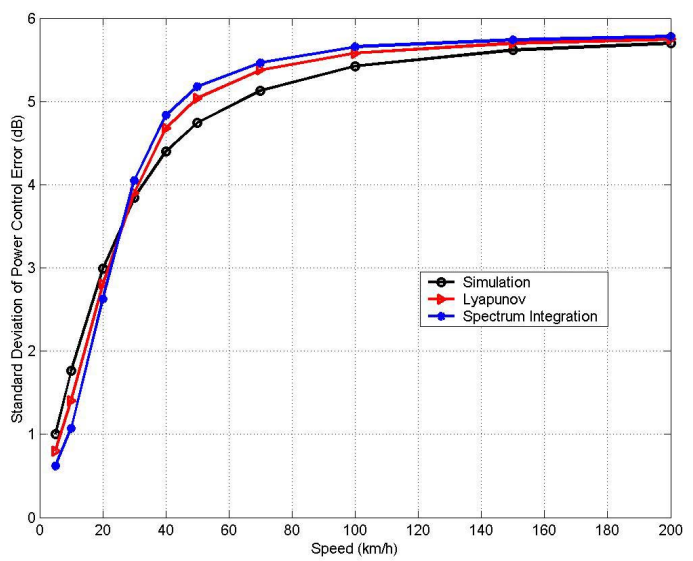

Figure 3: Standard Deviation of the Received PCE for Conv-CLPC The three curves (or their PCE standard deviations) present the common characteristics to converge towards the standard deviation value of the Rayleigh channel, $\left(\sigma_{A}\right.$ $=5.57 \mathrm{~dB}$ ) for high mobile velocity. This explains that for high doppler frequency the CLPC cannot track efficiently the fast fading [4]. Notice, same type of convergence was observed for shadowing [3]. The three plots match each other closely but the imprecision between the analytical methods and the simulation method is due to the assumptions made by the analytical methods as well as exact accuracy of the simulation method. The analytical methods produce these results much faster than the simulation [1]. Therefore, to analyze the CLPC for different parameters, such as step size, delay effect or others very rapidly, the analytical method becomes therefore a powerful tool compared to the lengthy simulations.

\section{Quantized step size power control}

So far only the performance of the fixed-step size power control algorithm was evaluated. It has been proved that the proposed analytical model performance is relatively close to the simulation performance. We can therefore optimise the existing algorithm (conventional CLPC) via the analytical model.

We have implemented and analysed the process of quantization in the power control algorithm. The power control error is now quantized, so that the step size can adapt to an exact value. The quantizer consists of $2^{b}$ levels, where $b$ is the number of bits used for a power control command. The degradation in performance originates from the delay introduced in the loop, since now the power control command consists of more than one bit; a delay is introduced in the loop. In general, time delays are primarily of two kinds. First there is a delay due to the time to measure and report the measurements to the algorithm and secondly there is a time delay due to the time it takes before the computed power level is actually used in the transmitter. Here we consider mainly the second one.

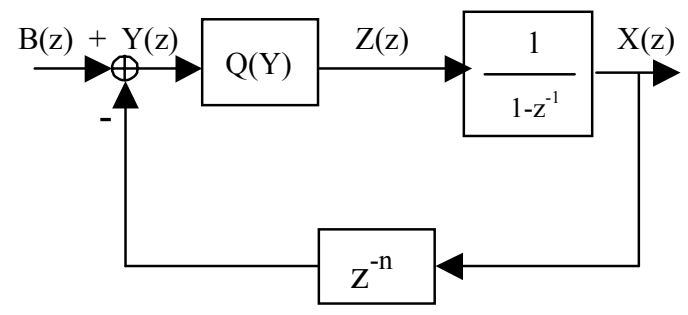

Figure 4: Block diagram for the Quantized step size power control algorithm.

We now assume that a quantizer replaces the hard limiter and the new state equation (deriving from Eq.2) is:

$X(n+1)=X(n)+Q(Y(n-k))$

Eq 15

, where $Q($.$) denotes the operation of quantization$ process. The PCE delayed by k power control commands (or slots in UMTS standard) is expressed as

$Y(n-k)=B(n-k)-X(n-k) \quad$ Eq 16

For the analysis of the closed loop presented in Figure 4, the statistical approach presented in [8] is adopted. The block diagram of Figure 5 is the corresponding mathematical model. It is assumed that the quantization error is random in nature and that it is added to the original signal as noise.

The error $\left\{e_{q}(n)\right\}$ is a stationary white noise sequence uniformly distributed in $[-\Delta / 2, \Delta / 2]$, where $\Delta$ is the quantizer step size.

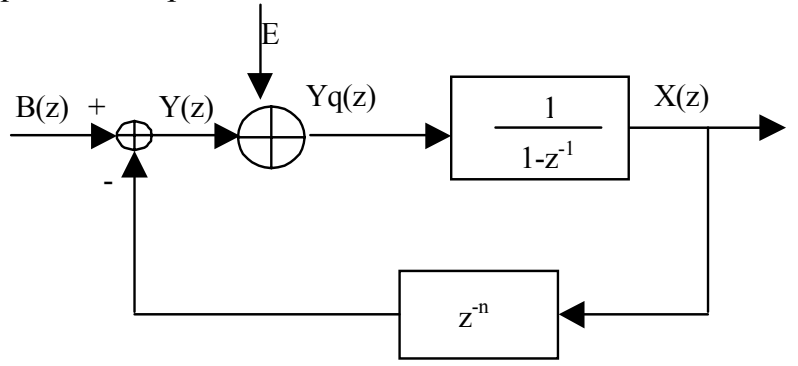

Figure 5: Mathematical model of Quantized step size power control algorithm

In other words the error samples are uncorrelated and the error sequence $\left\{e_{q}(n)\right\}$ is uncorrelated with the signal sequence. The noise power is then given by

$P_{n}=\sigma_{E}^{2}=\int_{-\Delta / 2}^{\Delta / 2} e^{2} p(e) d e=\frac{\Delta^{2}}{12}$

Eq 17

, where $\Delta=\frac{R}{2^{b}}$ and $R$ is the range of the quantizer.

Since the system depicted in Figure 5 is linear, the variance of the power control error can be found in the same way used in Eq.5:

$\sigma_{Y}^{2}=\frac{1}{2 \pi} \int_{-\pi}^{\pi}\left|H_{Y B}\left(e^{j \omega}\right)\right|^{2} P_{B}(\omega) d \omega+\frac{1}{2 \pi} \sigma_{E}^{2} \int_{-\pi}^{\pi}\left|H_{Y E}\left(e^{j \omega}\right)\right|^{2} d \omega$

Eq 18

and the new transfer functions are:

$H_{Y B}\left(e^{j \omega}\right)=\frac{e^{j \omega}-1}{e^{j \omega}-1+e^{-j \omega(n-1)}}$

$H_{Y E}\left(e^{j \omega}\right)=-\frac{e^{-j \omega n}}{e^{-j \omega n}+1-e^{-j \omega}}$ 
The spectrum of the Rayleigh channel is given by $P_{B}(\omega)$ (Eq.14) and considered for these channel values $\left(\sigma_{A}=5.57 \mathrm{~dB}, f_{c}=2 \mathrm{GHz}, \quad T=0.667 \mathrm{~ms}\right)$. Some interesting results regarding the effect of the quantizer range, number of bits used and delays in the performance of the CLPC have been obtained.

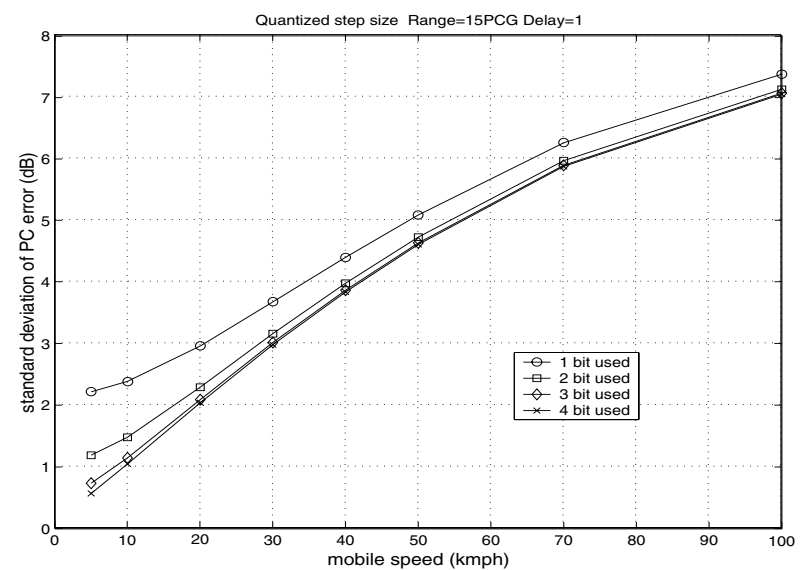

Figure 6: Effect of number of bits used

Figure 6 shows the effect of the number of bits used in the quantization process on the standard deviation of the power control error. Figure 7 and Figure 8 illustrate the effect of the range and loop delay respectively.

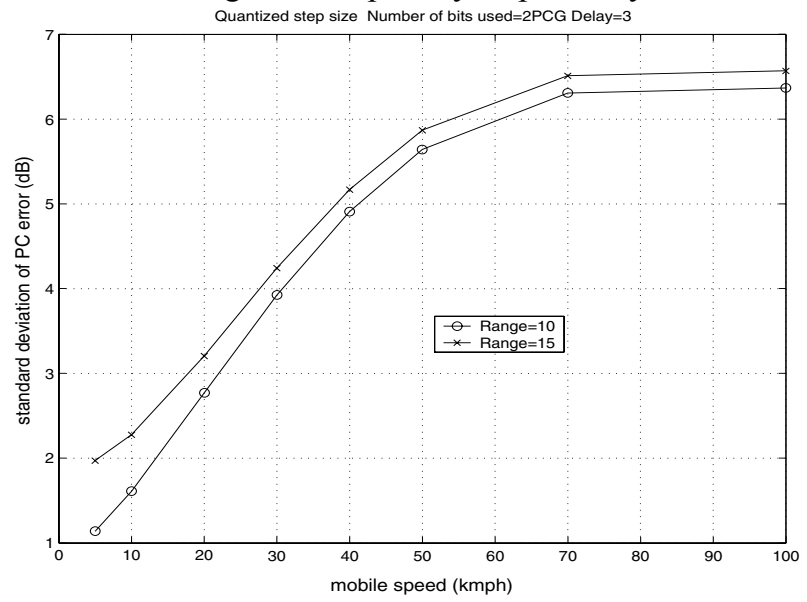

Figure 7: Effect of Quantizer input range

The observation of the Figures $6,7 \& 8$ helps the optimisation of the Quantizer parameters, namely range and number of levels-bits used, as well as in the visualization of the loop delay effect, which is unavoidable since now more than one bits per power control command are transmitted. As the input range $R$ increases, the power control algorithm shows worse performance. This is expected since it is proved from Eq 17 that the noise power is proportional to $R$.

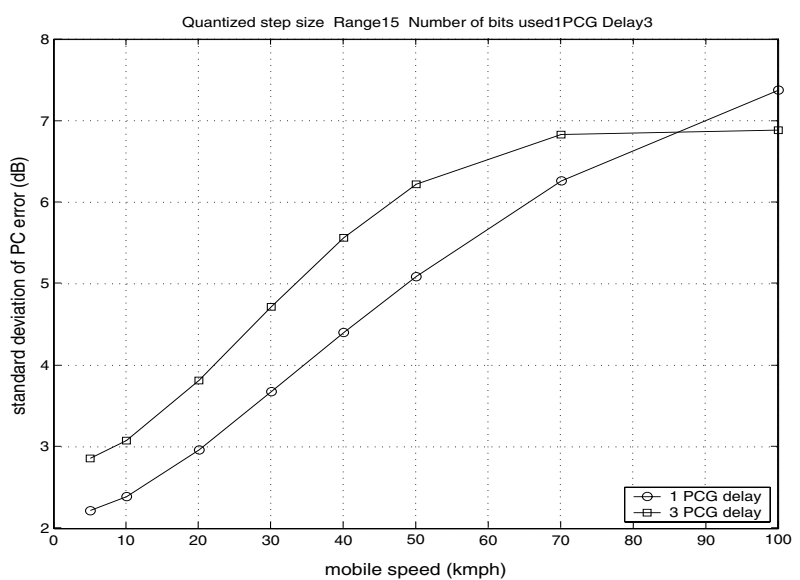

Figure 8: Effect of delay

As the number of bits $b$ used increase, the power control algorithm shows better performance. This is expected since it is proved from Eq 17 that the noise power is inversely proportional to $b$. However there is no point in using a lot of quantization levels to improve performance. Three bits are enough as shown in Figure 6. From this point, the increase in performance does not justify the delay imposed. Since more than one bit are now used for the power control command transmission, there is an unavoidable delay in the update of the mobile power. It should be expected that as the delay increases, the performance of the power control scheme gets worse (Figure 8), since the channel gain is badly tracked.

Before to proceed further, we have fixed some of these parameters to analyse the credibility of this method versus the simulation.

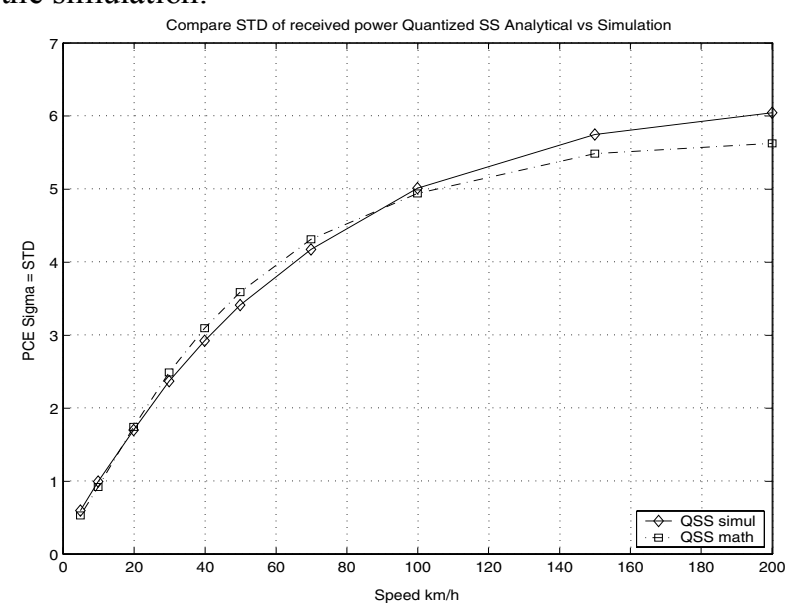

Figure 9: Quantized CLPC Analytical vs Simulation method

From the Figure 9 it can be seen that the results obtained analytically are verified by those obtained via simulation. In this latter each power control command consists of three bits (therefore the step size can have eight different values) and the range is fixed to $10 \mathrm{~dB}$.

The PDF of the received power at the BS (represented by QSS simul in Figure 9) for different MS velocity is also drawn in the Figure 10. 


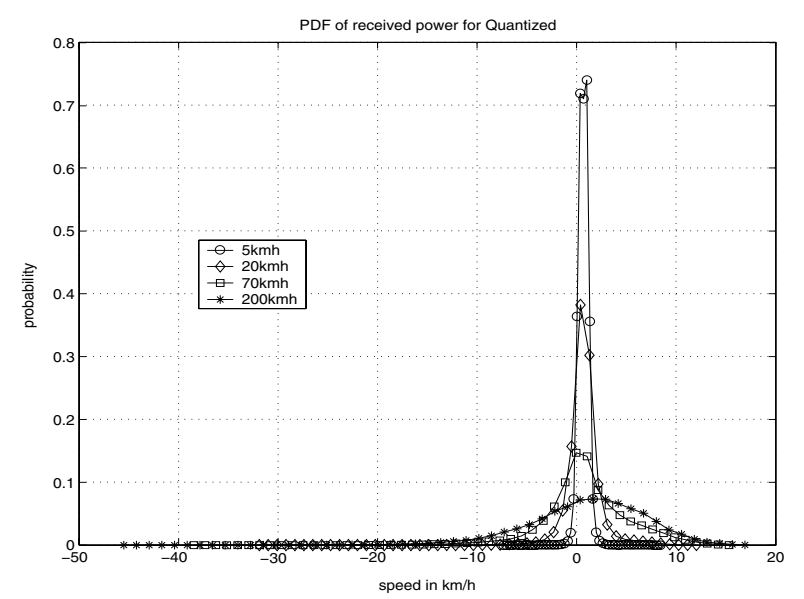

Figure 10: PDF of Quant-CLPC

Another important conclusion comes from the comparison of the fixed-step power control algorithm against the proposed quantized algorithm. For a better performance comparison of both algorithms, we have gathered the results of Figure $3 \& 9$ in Figure 11.

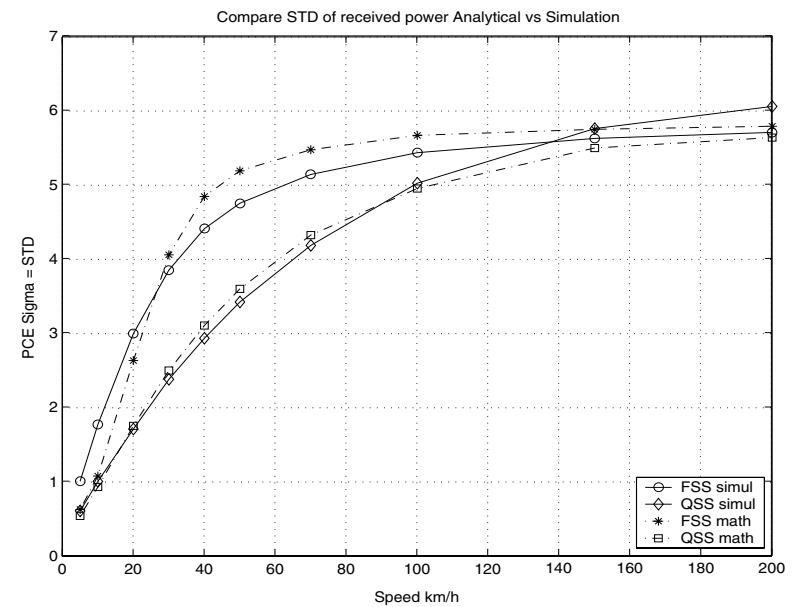

Figure 11: Fixed-step vs Quantized-step algorithms

In almost all cases the quantized-step scheme (QSS) outperforms the fixed-step scheme (FSS). Since the quantized step size can take a value closer to the accurate value required by the BS to change the MS transmit power, it is obvious that this algorithm performs better than the conventional method. Note, that in this performance the delay produced due to the extra number of bits used, is not affecting the gain obtained.

However, as the mobile speed increases (from $10 \mathrm{~km} / \mathrm{h}$ to $100 \mathrm{~km} / \mathrm{h}$ ) and the fading becomes faster, the fixed stepsize is not enough to compensate for the fading since bigger steps are needed [4]. In this case the quantized step size performs better since it can take bigger values as well. Nevertheless, above $100 \mathrm{~km} / \mathrm{h}$, the rate of the change in the fast fading is too high, and the error received at the BS is very close to the error due to the Rayleigh fading std. But at this speed, techniques such as interleaving are expected to improve the system performance.

\section{Conclusion}

This paper has analytically evaluated the performance of the CLPC used in a CDMA system. Since the evaluation is usually done by lengthy simulations, the analytical method provides faster results. We have shown the wellknown difficulty to evaluate this expression for the case of fast fading. We have proposed a fully analytical method under fast fading, which provides close results compared to the simulated ones. This method allowed us to analyse the quantized step size algorithm and its performance was also analytically evaluated versus the simulation. We have shown that the quantized method is an interesting tool to optimise the performance. For an optimum performance, a compromise must be made between the number of quantization levels and the delay imposed. An improvement to this work would be the use of differential quantization in order to reduce adaptively the dynamic range and consequently the number of bits to transmit the power control command. Also there have been many analysis on the CLPC based on adaptive step size, but any fast changes in the transmit power could affect the interference stabilisation (or convergence) at the system level. The proposed model is applicable to any CDMA systems regarding an appropriate choice of the channel, such as Rician channel for Satellite Communications based on CDMA systems.

\section{References}

[1] Sim M.L., Gunawan E., Soong B-H., Soh C-B., " Performance Study of Close-Loop Power Control Algorithms for a Cellular CDMA System", IEEE Trans., VT-48, No.3, 911-921, 1999

[2] Viterbi, A. and Padovani, R. 'Implication of Mobile Cellular CDMA', IEEE Communications Magazine, pp. 38-41, December 1992

[3] L. Song, N. Mandayam, and Z. Gajic, " Analysis of an Up/Down Power Control Algorithm for the CDMA Reverse Link under Fading ", IEEE JSAC Wireless Series, vol. 19, No. 2, pp. 277-286, February 2001

[4] S.Nourizadeh, P.Taaghol, R.Tafazolli, “A Novel Closed-Loop Power Control for UMTS", 3G Mobile Communication Technologies Conf. IEE, pp56-59, March 2000.

[5] Gudmundson, M 'Correlation Model for Shadow Fading in Mobile Radio Systems', Electronics Letters, vol. 27, pp.2145-2146, November 1991

[6] M. Patzold, "A Deterministic Method for the Derivation of a Discrete WSSUS Multipath Fading Channel Model", submitted to European Transactions on Telecommunications and Related Technologies, 1994.

[7] A. A. Hutter, "A Simple Smart Antenna Approach to Reduce the Error Floor for Mobile OFDM Systems," International conference on Telecommunications ICT 2000.

[8] Proakis, J.G and Manolakis, D.G 'Digital Signal Processing, Principles, Algorithms and Applications', Prentice Hall International, 1996 